\title{
A Two-Dimensional Linear Bicharacteristic FDTD Method
}

\author{
John H. Beggs \\ NASA/Langley Research Center \\ Electromagnetics Research Branch \\ Mail Stop 490 \\ Hampton, VA 23681
}

\section{INTRODUCTION}

The linear bicharacteristic scheme (LBS) was originally developed to improve unsteady solutions in computational acoustics and aeroacoustics [1]-[7]. The LBS has previously been extended to treat lossy materials for one-dimensional problems [8]. It is a classical leapfrog algorithm, but is combined with upwind bias in the spatial derivatives. This approach preserves the time-reversibility of the leapfrog algorithm, which results in no dissipation, and it permits more flexibility by the ability to adopt a characteristic based method. The use of characteristic variables allows the LBS to include the Perfectly Matched Layer boundary condition with no added storage or complexity. The LBS offers a central storage approach with lower dispersion than the Yee algorithm, plus it generalizes much easier to nonuniform grids. It has previously been applied to two and three-dimensional freespace electromagnetic propagation and scattering problems [3], [6], [7]. This paper extends the LBS to the two-dimensional case. Results are presented for point source radiation problems, and the FDTD algorithm is chosen as a convenient reference for comparison.

\section{TWO-DIMENSIONAL IMPLEMENTATION}

Maxwell's equations in bicharacteristic form for linear, homogeneous and lossy media in the twodimensional TM case (taking $\partial / \partial z=0$ ) are

$$
\begin{aligned}
& \frac{\partial}{\partial t}\left(D_{z} \mp \frac{1}{c} H_{y}\right) \pm c \frac{\partial}{\partial x}\left(D_{z} \mp \frac{1}{c} H_{y}\right)+\frac{\sigma}{\epsilon} D_{z}-\frac{\sigma^{*}}{\mu c} H_{y}+\frac{\partial H_{x}}{\partial y}=0 \\
& \frac{\partial}{\partial t}\left(D_{z} \pm \frac{1}{c} H_{x}\right) \pm c \frac{\partial}{\partial y}\left(D_{z} \pm \frac{1}{c} H_{x}\right)+\frac{\sigma}{\epsilon} D_{z}+\frac{\sigma^{*}}{\mu c} H_{x}-\frac{\partial H_{y}}{\partial x}=0
\end{aligned}
$$

where $c=1 / \sqrt{\mu \epsilon}$, and the electric and magnetic conductivities are given by $\sigma$ and $\sigma^{*}$, respectively. The characteristic variables are defined as $P=D_{z}-\frac{1}{c} H_{y}, Q=D_{z}+\frac{1}{c} H_{y}, R=D_{z}+\frac{1}{c} H_{x}$, $S=D_{z}-\frac{1}{c} H_{x}$ to represent the $\pm x$ and $\pm y$ propagating solutions, respectively. Equations (1)-(2) can be rewritten more concisely as

$$
\begin{aligned}
& \frac{\partial P}{\partial t}+c \frac{\partial P}{\partial x}+\frac{a}{2} P+\frac{b}{2} Q+\frac{\partial H_{x}}{\partial y}=0 \\
& \frac{\partial Q}{\partial t}-c \frac{\partial Q}{\partial x}+\frac{b}{2} P+\frac{a}{2} Q+\frac{\partial H_{x}}{\partial y}=0 \\
& \frac{\partial R}{\partial t}+c \frac{\partial R}{\partial y}+\frac{a}{2} R+\frac{b}{2} S-\frac{\partial H_{y}}{\partial x}=0 \\
& \frac{\partial S}{\partial t}-c \frac{\partial S}{\partial y}+\frac{b}{2} R+\frac{a}{2} S-\frac{\partial H_{y}}{\partial x}=0
\end{aligned}
$$

where $a=\sigma / \epsilon+\sigma^{*} / \mu$ and $b=\sigma / \epsilon-\sigma^{*} / \mu$. To develop the discretized algorithm for a twodimensional system, the stencils of Figure 1 are proposed for the 2D LBS. The upwind bias nature of these stencils is clearly evident. We discretize time and space as $t=n \Delta t, x=i \Delta x, y=j \Delta y$, and we also know that $H_{x}=c(R-S) / 2$ and $H_{y}=c(Q-P) / 2$. Using the stencils shown in Figure 1, the resulting finite difference equations for (3)-(6) are

$$
(1+a \Delta t) P_{i+1 / 2, j}^{n+1}=P_{i-1 / 2, j}^{n-1}+\left(1-2 \nu_{x}\right)\left(P_{i+1 / 2, j}^{n}-P_{i-1 / 2, j}^{n}\right)-b \Delta t Q_{i+1 / 2, j}^{n}-
$$




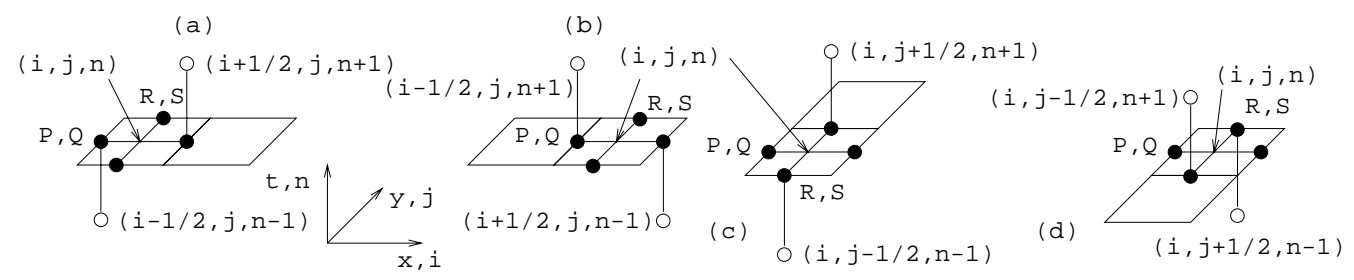

Fig. 1. Two-dimensional upwind leapfrog computational stencils for (a) $+x$; (b) $-x$; (c) $+y$ and (d) $-y$ propagating characteristics.

$$
\begin{aligned}
& \nu_{y}\left(R_{i, j+1 / 2}^{n}-R_{i, j-1 / 2}^{n}\right)+\nu_{y}\left(S_{i, j+1 / 2}-S_{i, j-1 / 2}^{n}\right) \\
(1+a \Delta t) Q_{i-1 / 2, j}^{n+1}= & Q_{i+1 / 2, j}^{n-1}-\left(1-2 \nu_{x}\right)\left(Q_{i+1 / 2, j}^{n}-Q_{i-1 / 2, j}^{n}\right)-b \Delta t P_{i-1 / 2, j}^{n}- \\
& \nu_{y}\left(R_{i, j+1 / 2}^{n}-R_{i, j-1 / 2}^{n}\right)+\nu_{y}\left(S_{i, j+1 / 2}-S_{i, j-1 / 2}^{n}\right) \\
(1+a \Delta t) R_{i, j+1 / 2}^{n+1}= & R_{i, j-1 / 2}^{n-1}+\left(1-2 \nu_{y}\right)\left(R_{i, j+1 / 2}^{n}-R_{i, j-1 / 2}^{n}\right)-b \Delta t S_{i, j+1 / 2}^{n}- \\
& \nu_{x}\left(P_{i+1 / 2, j}^{n}-P_{i-1 / 2, j}^{n}\right)+\nu_{x}\left(Q_{i+1 / 2, j}-Q_{i-1 / 2, j}^{n}\right) \\
(1+a \Delta t) S_{i, j-1 / 2}^{n+1}= & S_{i, j+1 / 2}^{n-1}-\left(1-2 \nu_{y}\right)\left(S_{i, j+1 / 2}^{n}-S_{i, j-1 / 2}^{n}\right)-b \Delta t R_{i, j-1 / 2}^{n}- \\
& \nu_{x}\left(P_{i+1 / 2, j}^{n}-P_{i-1 / 2, j}^{n}\right)+\nu_{x}\left(Q_{i+1 / 2, j}-Q_{i-1 / 2, j}^{n}\right)
\end{aligned}
$$

where $P_{i, j}^{n}$ denotes the value for $P$ at grid point $(i, j)$ and time level $n$ and $\nu_{x}=c \Delta t / \Delta x, \nu_{y}=$ $c \Delta t / \Delta y$ are the $x$ and $y$ Courant numbers. Note that the differences are taken with respect to the cell center, i.e. the coordinate $(i, j)$ is located at the center of the cell. The procedure for the TE polarization is similar, except the characteristic variables are defined by $P=D_{y}+\frac{1}{c} H_{z}$, $Q=D_{y}-\frac{1}{c} H_{z}, R=D_{x}-\frac{1}{c} H_{z}$, and $S=D_{x}+\frac{1}{c} H_{z}$. The update equations are almost identical, differing only in signs of the cross derivative terms. The 2D LBS includes the PML boundary condition in its formulation without any additional storage or complexity. Starting with the bicharacteristic Maxwell's equations in free space [(1)-(2) with $\sigma=\sigma^{*}=0$ ], it can be shown using a complex coordinate transformation PML approach that the resulting equations are identical to (3)-(6). A full derivation of these equations is outlined in [9]. Thus, the LBS automatically implements the PML when combined with the standard PML conductivity profiles: linear, quadratic or geometric [10].

\section{FOURIER ANALYSIS}

Various Fourier analyses of the two-dimensional LBS have already been completed [5], [6], [7]; therefore, only the important results and conclusions from these previous analyses will be reviewed in this paper. Most of the information presented is summarized from [5]. The stability condition for the 2D LBS is $\nu_{x}, \nu_{y} \leq$ $1 / 2$. Although this stability limit is more restrictive than the standard 2D FDTD method, it is not particularly troublesome because many FDTD simulations use a Courant number of $1 / 2$ for improved accuracy. For a Courant number $\nu=0.4$ and propagation angle of $45^{\circ}$, the numerical dispersion decreases smoothly with increasing grid resolution as shown in Figure 2. From this figure, we see that the LBS has approximately $1 / 2$ the phase error as FDTD. Generally, the

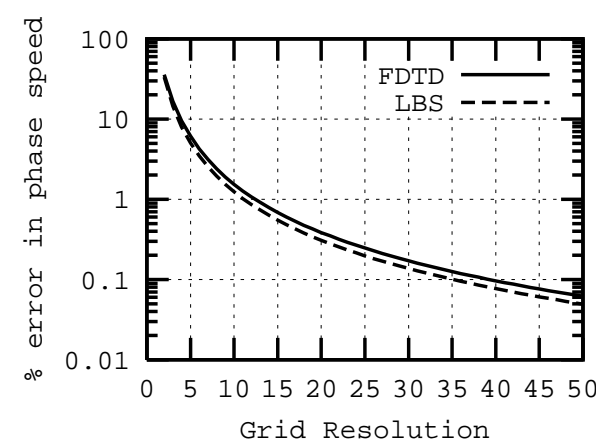

Fig. 2. Phase speed error versus grid resolution $N$ for FDTD method and LBS with $\nu=0.4$ and $\alpha=45^{\circ}$

dispersion error for the LBS grows as $\nu \rightarrow 0$. When $\nu=1 / 2$, numerical dispersion is zero along the 
coordinate axes and is maximum at $45^{\circ}$ as shown in Figure 3 for a grid resolution $N=10$ cells $/ \lambda$. The optimal Courant number for the LBS is $1 / 2$. This Courant number offers much lower dispersion for most all propagation angles except those near a $45^{\circ}$ vector. For $\nu<1 / 2$, numerical dispersion decreases as both grid resolution and Courant number are increased. Typically, LBS dispersion is at least $1 / 2$ that of FDTD, and can be much lower in many instances.

\section{NumERICAL RESUlts}

To demonstrate the 2D LBS, we consider various canonical problems. First, we inject an incoming plane wave on the outer boundaries using the LBS, and let the algorithm propagate the signal through the grid using a total field formulation. This is done by specifying the incoming characteristic variable $(P, Q, R$ or $S)$ on the appropriate outer boundary. For example, on the left $x$ boundary, $P$ is specified for all $j$ coordinates at $i=1$. We use a $71 \times 71$ free space grid, with a $\Delta x=\Delta y=1$ $\mathrm{cm}$, which has a time step of $\Delta t=16.67 \mathrm{ps}$ and the incident wave is a Gaussian pulse with FWHM of 35 time steps (or $0.58 \mathrm{~ns}$ ). We specify the incidence angle as $180^{\circ}$, and the electric field after 160 time steps is shown in Figure 4. Similar results can be obtained with other incidence angles. It is clear

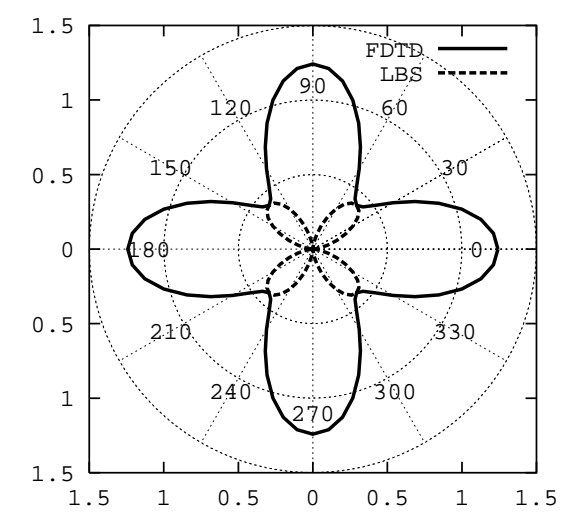

Fig. 3. Phase speed error versus grid propagation angle $\alpha$ for FDTD method and LBS with $\nu=$ $1 / 2$ and $N=10$. that the LBS easily allows specification of incoming plane waves in its fundamental algorithm.

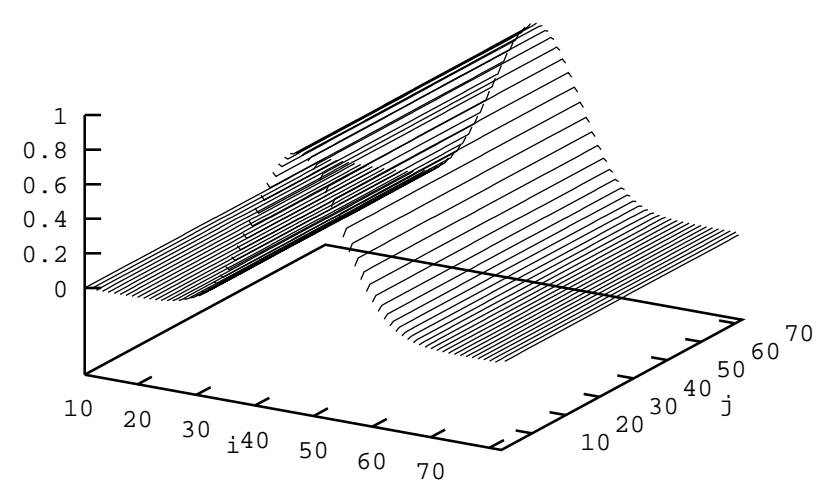

Fig. 4. Propagating plane wave injected on outer grid boundaries at $180^{\circ}$ incidence.

Next we move on to radiation from a point source in free space. This problem demonstrates that the algorithm can easily treat spherical waves and it also tests the PML boundary condition. Two concurrent grids are used in this problem, each having a cell size of $1 \mathrm{~mm}$. The first is a small test grid of size $101 \times 101$ cells with an additional 10 cell PML boundary condition. This grid is centered within a large $501 \times 501$ grid, and the point source is located at the center of both computational grids. The time step is $3.3 \mathrm{ps}$, and an electric field point source is located at the center of both grids and the total number of time steps is truncated at 512, to allow no reflection from the large grid outer boundaries to reach the field sampling points. The inner grid is terminated with PML for both FDTD and the LBS, and the large grid is terminated with a second-order Liao boundary condition for FDTD and a characteristic based boundary condition for the LBS. The electric field is sampled at the same two locations in both grids, which are located 30 cells in the $+x$ direction from the point source and then \pm 30 cells in the $y$ direction in the smaller grid. The point source is located in the smaller grid at grid point $(61,61)$ and the two sample points are $(61,91)$ and $(61,31)$. Figure 5 shows the electric field at the upper sample point $(61,91)$ in the smaller test grid (with PML) for point source radiation in free space. Note the agreement is excellent, and there are no reflections from the outer boundary due to the PML boundary condition. Similar results were observed at the lower sample point $(61,31)$. Next, we simulate the same point source radiation problem, but turn off the PML boundary condition and instead use the characteristic boundary condition. Figure 6 shows the results using for the LBS with and without the PML boundary condition. Note the reflections from the outer boundary are clearly visible for the no PML case. 


\section{CONCLuSions}

This paper has extended the Linear Bicharacteristic Scheme for computational electromagnetics to the two-dimensional case. It was demonstrated that the LBS has several distinct advantages over conventional FDTD algorithms. First, the LBS typically has much lower dispersion than the FDTD method. The LBS can also be made to have zero dispersion error in certain instances. Second, the LBS provides the PML boundary condition as part of the fundamental scheme, and does not require additional storage or computational complexity for the PML. Finally, the upwind biasing provides a more flexible generalization to unstructured grids, and for subgrid implementations. The two-dimensional LBS can easily treat dielectric and magnetic materials, and a dielectric surface boundary condition was implemented in a separate report [9]. However, validation of the 2D LBS for dielectric and magnetic materials was not explored in the present work and should be the subject of future articles. The LBS appears to be a very promising alternative to a conventional FDTD algorithm for many applications. Higher order schemes are available for the 2D case [5]. Additional research and development is required to explore the full potential of this new technique.

\section{REFERENCES}

[1] J. P. Thomas and P. L. Roe, "Development of non-dissipative numerical schemes for computational aeroacoustics," AIAA, 1993, paper number 93-3382-CP.

[2] P. Roe, "Linear bicharacteristic schemes without dissipation," Tech. Report 94-65, ICASE, NASA/Langley Research Center, Hampton, VA, 1994

[3] B. Nguyen and P. Roe, "Application of an upwind leap-frog method for electromagnetics," in Proc. 10th Annual Review of Progress in Applied Computational Electromagnetics, Monterey, CA, March 1994, Applied Computational Electromagnetics Society, pp. 446-458.

[4] J. P. Thomas, C. Kim and P. Roe, "Progress toward a new computational scheme for aeroacoustics," in AIAA 12th Computational Fluid Dynamics Conference. AIAA, 1995.

[5] J. P. Thomas, An Investigation of the Upwind Leapfrog Method for Scalar Advection and Acoustic/Aeroacoustic Wave Propagation Problems, Ph.D. thesis, University of Michigan, Ann Arbor, MI, 1996.

[6] B. Nguyen, Investigation of Three-Level Finite-Difference Time-Domain Methods for Multidimensional Acoustics and Electromagnetics, Ph.D. thesis, University of Michigan, Ann Arbor, MI, 1996.

[7] C. Kim, Multidimensional Upwind Leapfrog Schemes and Their Applications, Ph.D. thesis, University of Michigan, Ann Arbor, MI, 1997.

[8] John H. Beggs, The Linear Bicharacteristic Scheme for Electromagnetics, NASA/Langley Research Center, Hampton, VA, May 2001, NASA-TM-2001-210861.

[9] John H. Beggs, A Two-Dimensional Linear Bicharacteristic Scheme for Electromagnetics, NASA/Langley Research Center, Hampton, VA, Technical Memorandum, in preparation.

[10] A. Taflove, Ed., Advances in Computational Electrodynamics: The Finite-Difference Time-Domain Method, Artech House, Boston, MA, 1998.

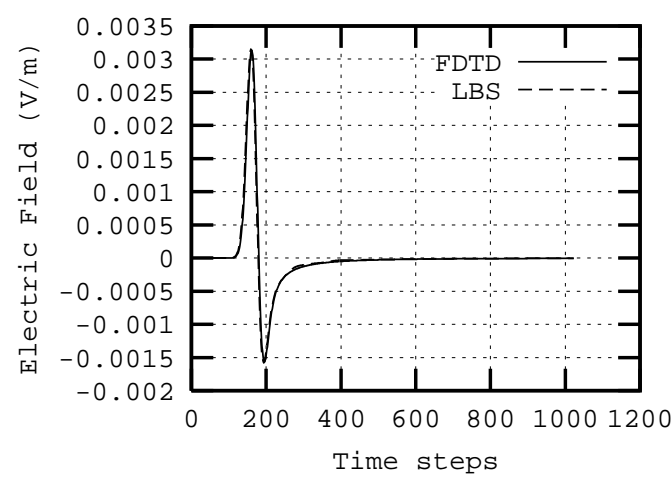

Figure 5: Sampled electric field due to radiation from a point source in free space.

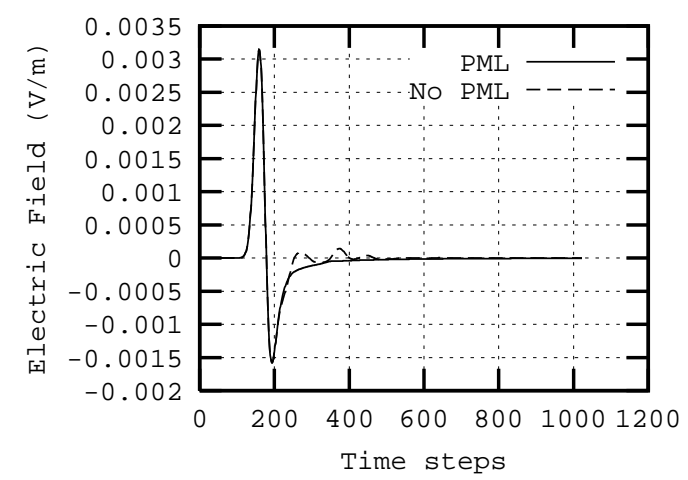

Figure 6: Sampled electric field due to radiation from a point source in free space for LBS with and without PML boundary condition. 